\title{
Spatiotemporal variability of abundance size-spectra in streams across North America
}

\author{
Justin Pomeranz ${ }^{1}$, James R. Junker ${ }^{2}$, and Jeff Wesner ${ }^{3}$ \\ ${ }^{1}$ University of Canterbury \\ ${ }^{2}$ Affiliation not available \\ ${ }^{3}$ University of South Dakota
}

August 27, 2021

\begin{abstract}
Accepted version of this manuscript can be found here: https://doi.org/10.1111/gcb.15862

The distribution of abundance and biomass within ecological communities is related to trophic transfer efficiency from prey to predators. While it is considered to be one of the few consistent patterns in ecology, spatiotemporal variation of this relationship across continental-scale environmental gradients is unknown. Using a database of stream communities collected across North America (18-68 $\mathrm{N}$ latitude, -4 to $25^{\circ} \mathrm{C}$ mean annual temperature) over 3 years, we constructed 162 mass-abundance relationships (i.e. size spectra). Size-spectra slopes declined (became steeper) with increasing temperature. However, the magnitude of change was relatively small, with median slopes changing from -1.2 to -1.3 across a $29^{\circ} \mathrm{C}$ range in mean annual temperature. In contrast, total community biomass increased 3-fold over the temperature gradient. Our study suggests strong conservation of abundance size-spectra in streams across broad natural environmental gradients. This supports the emerging use of size-spectra deviations as indicators of ecosystem health.
\end{abstract}

\section{Spatiotemporal variability of abundance size-spectra in streams across North America}

Justin P. F. Pomeranz ${ }^{1 *}$, Jeff S. Wesner ${ }^{1}$

${ }^{1}$ Department of Biology, University of South Dakota.

* corresponding author, Justin Pomeranz

Email:jfpomeranz@gmail.com; jeff.wesner@usd.ed

ORCIDs: Justin Pomeranz: 0000-0002-3882-7666; Jeff Wesner: 0000-0001-6058-7972

Running Title: NEON Stream Size Spectra

\section{Keywords}

Biomass distribution, size spectra, community structure, allometric scaling, body size, biogeography, macroecology, freshwater ecology, macroinvertebrates

Type of article: Letters

Number of words in:

Abstract: 145

Main text: 3625

Number of references: 45 


\title{
Number of figures: 3 \\ Number of tables: 2
}

Corresponding author: Justin Pomeranz, jfpomeranz@gmail.com, cell: 720.212.9517, Mailing address: University of South Dakota, Department of Biology, 401 East Clark St, Vermillion, SD, USA 57069

Author contributions: JP designed the study, collected the data, performed the modelling work, made the figures and wrote the first draft of the manuscript, JW performed modelling work, made the figures, and all authors contributed substantially to revisions.

Data accessibility: Upon article acceptance, all data and code used in the present study will be archived in Data Dryad and Zenodo, respectively.

\begin{abstract}
The distribution of abundance and biomass within ecological communities is related to trophic transfer efficiency from prey to predators. While it is considered to be one of the few consistent patterns in ecology, spatiotemporal variation of this relationship across continental-scale environmental gradients is unknown. Using a database of stream communities collected across North America (18-68 $\mathrm{N}$ latitude, -4 to $25^{\circ} \mathrm{C}$ mean annual temperature) over 3 years, we constructed 162 mass-abundance relationships (i.e. size spectra). Sizespectra slopes declined (became steeper) with increasing temperature. However, the magnitude of change was relatively small, with median slopes changing from -1.2 to -1.3 across a $29^{\circ} \mathrm{C}$ range in mean annual temperature. In contrast, total community biomass increased 3 -fold over the temperature gradient. Our study suggests strong conservation of abundance size-spectra in streams across broad natural environmental gradients. This supports the emerging use of size-spectra deviations as indicators of ecosystem health.
\end{abstract}

\section{Introduction}

Many fundamental aspects of an organism's biology are controlled by body size, including metabolic rate, life history characteristics, diet breadth, and trophic position (Brown et al. 2004; Woodwardet al. 2005; White et al. 2007). Communities, particularly in aquatic environments, are often size structured and characterized by a strong negative relationship between the abundance or biomass of individuals and body size, known as the size spectrum (White et al. 2007; Blanchard et al. 2009). The slope of this relationship is related to trophic transfer efficiency (Trebilco et al. 2013; Sprules \& Barth 2015). Therefore, understanding the distribution of biomass within communities connects individual- and population-level traits to community structure, function, and ecosystem dynamics (Dossenaet al. 2012; O'Gorman et al. 2012; Yvon-Durocher \& Allen 2012; Trebilco et al. 2013).

Size spectra are one of the few well documented organizing principles in ecology. A large body of literature has demonstrated the consistency of size-spectra relationships in diverse ecosystems (Jennings \& Blanchard 2004; Trebilco et al. 2013; Blanchard et al. 2017; Mazurkiewicz et al. 2019, 2020). A strongly negative relationship between the abundance or biomass of individuals for a given body size range has been consistently documented in aquatic communities, to the point that size-spectra parameters have been recommended as a potential "universal" indicator of ecological health (Petchey \& Belgrano 2010).

However, while estimated slope coefficients are universally consistent in sign (i.e. always negative), their specific values can vary in response to both natural and anthropogenic environmental drivers (Yvon-Durocher et al. 2011; Dossena et al. 2012; O'Gormanet al. 2012; McGarvey \& Kirk 2018; Pomeranz et al. 2019). For example, because metabolic rates increase with both body size and temperature, environmental warming may have asymmetrical effects on community structure and biomass distributions (Brown et al. 2004; Brose et al. 2012). Bergmann's and James' rule predict that warmer regions will tend to have smaller species, or smallersized populations within a species, respectively (Bergmann 1847; James 1970). Further, the temperature-size rule states that warmer temperatures cause smaller individual body sizes in ectotherm species (Atkinson 1994). Finally, small body size is a predicted response to global warming (Daufresne et al. 2009; Gardner et al. 2011). Therefore, temperature is hypothesized to be one of the main drivers of variation in size spectra 
(O'Gorman et al. 2012, 2017). Other anthropogenic impacts, including land use (Martínez et al. 2016), acid mine drainage (Pomeranz et al. 2019), as well as natural variation, including seasonal variation (McGarvey \& Kirk 2018), and resource subsidies (Perkins et al. 2018), have also caused slope estimates to vary.

While these and other studies suggest that environmental conditions can alter size spectra relationships, it is difficult to know how those effects scale up to broader patterns in size-spectra at the continental scale and across multiple years. A major limiting factor in large-scale studies of size spectra is the logistical challenge of obtaining consistently collected, processed, and analyzed data across a large spatiotemporal scale. As a result, empirical studies of size spectra share a fundamental limitation of small geographic scales and limited sampling through time (typically a single sample). To overcome this limitation, we estimated size spectra across stream sites in the National Ecological Observatory Network (NEON). We had two primary objectives in this study: 1) assess the broad geographical consistency of size spectra across North American streams, 2) test the hypothesis that size spectra vary as a function of temperature (measured as mean annual temperature).

\section{Materials and Methods}

Quantitative samples of benthic macroinvertebrates from NEON wadeable-stream sites from 2017-2019 were downloaded to determine size-spectra relationships (National Ecological Observatory Network 2020). NEON is a National Science Foundation (USA) funded program which collects standardized samples from 81 sites (24 streams) across a variety of terrestrial and aquatic ecosystems in North America. Repeat collections include automated instrument recordings and observational field sampling throughout the year, and data are available as open source data products (https://data.neonscience.org/home). The stream sites range across a broad environmental gradient (Figure 1), spanning from 18 to $68^{\circ} \mathrm{N}$ latitude (majority between 33 to $45^{\circ}$ $\mathrm{N}$ ), mean annual temperatures of -4 to $25^{\circ} \mathrm{C}$, and mean annual precipitation of 331 to $2530 \mathrm{~mm}$ (Table 1). Additionally, sites are in varying biomes and terrestrial plant communities, and subject to different local geologies and flow regimes.

Sites were sampled 1-4 times each year across the local growing season. Most sites had at least two years with three samples, except for COMO which only had one year with three samples (2018), and two samples collected in both 2017 and 2019. Additionally, three sites only had data available from a single collection in a single year; REDB in 2018, WALK and WLOU both in 2019. Details of sample collection and processing protocols are available at the NEON website. Briefly, macroinvertebrate samples were collected from a known area using the sampling method most suited to a site. Laboratory processing included subsampling for taxonomic identification and size class measurement (nearest $\mathrm{mm}$ ) and estimating the total count per sample. Estimated total counts were standardized to individuals per $\mathrm{m}^{2}$ by dividing by the area sampled (Chesney 2019). Macroinvertebrate size classes (mm) were converted to individual dry mass ( $\mathrm{M}$, in $\mathrm{mg}$ ) using published length-weight regression coefficients. Most (96\%) taxa had taxon-specific length-weight regressions and these were used for model development and assessment. Approximately $8 \%$ of the observations were flagged by NEON as being damaged, affecting their length measurements, and were removed from the data. The final data set included length measurements for $>82,000$ individuals and counts that totaled $>17$ million individuals.

Size spectra slopes have been estimated using a variety of methods, with binning methods being common in the published literature (White et al. 2008; Sprules \& Barth 2015; Edwards et al. 2017). However, recent comparative studies have shown binning methods to be inaccurate, providing biased slope estimates, and recommend using maximum likelihood methods (Edwards et al. 2017). We fitted body size data (dry weight estimated from length-weight regressions) of macroinvertebrates from each collection to a bounded power law distribution with a probability density function:

$$
f(x)=\frac{(b+1) x^{b}}{x_{\max }^{b+1}-x_{\min }^{b+1}}, \quad b \neq 1
$$




$$
f(x)=\frac{1}{\log x_{\max }-\log x_{\min }}, \quad b=1
$$

Where $x$ is body mass, $b$ is the scaling exponent (size spectra slope), and the distribution is bounded by the minimum $\left(x_{\min }\right)$ and maximum $\left(x_{\max }\right)$ body sizes observed in a collection. Maximum likelihood methods were used to estimate the exponent, $b$, using code modified from Edwards et al. (2017). Using these methods, a more negative bexponent represents a steeper slope of the size spectra. Slopes can be steeper due to either a relative increase in the abundance of small body sizes, or a relative decrease in the abundance of large body sizes, or a combination of the two. Regardless, steeper slopes represent a relatively smaller proportion of large individuals compared to small individuals within a community.

In addition to the size spectra slopes, we also examined how total community biomass varied across the collections. First, we multiplied the estimated biomass of individuals by their estimated density to calculate $\mathrm{mg}$ of dry mass per $\mathrm{m}^{2}$ for each sample within a collection ( $\mathrm{n}=8$ samples per collection). The 8 samples per collection were used as individual observations in the hierarchical Bayesian analyses (see Statistical Analyses ) .

Macroinvertebrates were generally sampled three times within the growing season from each site. However, due to the wide geographical distribution of sites, there was significant variation in the sample dates. For example, samples in Alaska (latitude 65-68 $\mathrm{N}$ ) were collected between May and August, whereas samples in Alabama (latitude $33^{\circ} \mathrm{N}$ ) were collected between February and November. Because we are interested in the effects of temperature on size spectra, we used the mean annual temperature in degrees Celsius for each site.

\section{Statistical Analyses}

To estimate the effect of temperature on size spectra slopes (bexponent) and standing stock community biomass, we fit separate hierarchical models with varying intercepts across sites and year (McElreath 2020). We chose a Bayesian approach because it easily incorporates prior information and hierarchical model structures (Hobbs \& Hooten 2015; Dietze 2017). Because the exponent $b$ is continuous and can be positive or negative, we used a Gaussian likelihood to model size spectra slopes. The predictor variable was mean annual temperature. To account for unexplained variation among sites and years, we included random intercepts of site and year. Priors for the intercept were set as Normal $(-1.5,1)$. This puts low prior probabilities on positive exponents and on exponents with extreme negative values (e.g., $<-4$ ), reflecting a wide range of possible values reported in the size spectra literature (White et al. 2007, Blanchard et al. 2009, Edwards et al. 2017). Prior distributions for the effect of mean annual temperature were set as Normal $(0,0.1)$, and Exponential (2) for sigma and the standard deviation of the random intercepts. To ensure that the prior distributions contained reasonable prior prediction but did not overwhelm the posterior inference, we used prior predictive simulation (see Supplemental Information, Fig. S1; Gabry et al. 2019).

For community biomass, we used a Gamma likelihood with a log link, because biomass is a continuous and positive measure (Hobbs \& Hooten 2015). The model structure for biomass was the same as the model structure for size spectra slopes. The prior for the intercept was set as Normal $(7,1)$. This reflects a prior expectation that community biomass values of $\sim 150$ to $\sim 9000 \mathrm{mg}$ dry mass $/ \mathrm{m}^{2}$ are reasonable, with a mean of $\sim 1000$. These point estimates are obtained by exponentiating, due to the log-link, the prior mean (i.e. 7 ) plus and minus two standard deviations $(7-2$ or $7+2)$. These values are compatible with the range of values reported in the literature (Benke et al.1984; Grimm 1988; Warmbold \& Wesner 2018). The remaining priors wereNormal $(0,0.1)$ for the slope, Exponential (2) for the standard deviation of the random intercepts, and Gamma $(0.01,0.01)$ for the shape parameter of the Gamma likelihood. As before, these priors were specified using prior predictive simulation (Supplemental Information, Fig. S1). Our prior and posterior distributions for parameter coefficients are plotted in SI Figures S2 and S3. A sensitivity analysis of our priors indicated our parameter coefficient estimates were robust to halving and doubling the SD value (Fig. S6). The full hierarchical model structure can be found in Supplemental Information S1. 
Models were specified using the brms package (Bürkner 2018) in R (R Development Core Team 2017), with posterior distributions derived using Hamiltonian Monte Carlo in rstan (Stan Development Team 2018). The models were run using 4 chains each with 6000 iterations, in which the first half were discarded as warmup, resulting in 12000 posterior draws. Convergence was checked by ensuring that all $\mathrm{r}$-hats were $<1.1$, and by visually assessing trace plots (Gelman \& Rubin 1992). To assess model fit, we used posterior predictive checks in which we simulated ten data sets from the posterior distribution and graphically compared them to the original data set (Gelman et al. 2013; Gabry et al. 2019; Figure S4 and S5). Strong visual discrepancies between the original data and simulated data indicate poor model fit (Gabry et al. 2019). In our simulations, both models produced data that were qualitatively similar to the original data, indicating good model fit (SI, Figures S4 \& S5).

Data availability

All of the data and $\mathrm{R}$ code to reproduce our results and figures will be archived upon article acceptance. The data will be archived in an appropriate repository (i.e., Dryad) and the code will be archived on Zenodo.

\section{Results}

\section{Size Spectra slopes}

The maximum likelihood estimates of the power law exponent (e.g., size spectra slopes) for individual collections ranged from -0.73 to -1.80 . The median of the posterior distribution of size spectra slopes from the hierarchical Bayesian model was -1.25 (95\% CrI (-1.39, -1.11); Table 2). Size-spectra slopes had a $98 \%$ probability of being negatively related to mean annual temperature (coefficient estimate $=-0.004,95 \% \mathrm{CrI}$ $(-0.007,0.0004)$; Table 2$)$, meaning that increasing temperature was associated with relatively fewer larger body sizes compared to small body sizes within a community (Figure 2A). However, the magnitude of this decline was small, with median slopes changing from -1.2 to -1.3 across the $29^{\circ} \mathrm{C}$ temperature gradient. This range was smaller than the among-sample range in measured slopes within sites (Figure 2A). Using the posterior predictive distribution from our model to estimate variation among sites, median slopes ranged from a low of -1.33 at site GUIL (mean annual temperature $=25^{\circ} \mathrm{C}$ ) to a high of -1.15 at site CARI (mean annual temperature $=-1^{\circ} \mathrm{C}$; Figure $\left.3 \mathrm{~A}\right)$.

\section{Community biomass}

Community biomass showed higher variation among sites (Figure 2B) with collection-specific medians ranging from 22.3 to $6,729 \mathrm{mg}$ dry mass per $\mathrm{m}^{2}$. The global median of the posterior samples from the hierarchical Bayesian model was 1,333 mg dry mass per $\mathrm{m}^{2}$ (95\% CrI $(247,10$ 929)). Community biomass had a $95 \%$ probability of being positively related to mean annual temperature (coefficient estimate $=0.04$ (CrI $(-0.008$, 0.09); Table 2). After exponentiating, this indicates that each unit increase in mean annual temperature causes an average of a $4 \%$ increase in biomass. From the posterior predictive distribution, median biomass values ranged from a low of $285 \mathrm{mg}$ dry mass per $\mathrm{m}^{2}$ at site CARI (mean annual temperature $=-1^{\circ} \mathrm{C}$ ) to a high of $11,309 \mathrm{mg}$ dry mass per $\mathrm{m}^{2}$ at site LEWI (mean annual temperature $=12^{\circ} \mathrm{C}$, Figure $3 \mathrm{~B}$ ).

\section{Discussion}

We analyzed the largest collection of size spectra relationships in stream communities that we are aware of, and we found that slope estimates varied in response to a broad temperature gradient. Size-spectra slopes represent the efficiency of energy transfer from small, abundant, individuals to fewer large predators (Trebilco et al. 2013) with clear implications for ecosystem functioning (O'Gormanet al. 2012; Cross et al. 2015). Shallow slopes indicate efficient transfer of energy by supporting a relatively higher proportion of larger individuals, while steeper slopes indicate inefficient energy transfer with relatively fewer large individuals. The steeper slopes reported here with increasing mean annual temperatures implies that warmer sites have fewer large individuals relative to the number of small individuals within a site compared to colder sites.

These results help to resolve previous uncertainty in how size spectra slopes scale with temperature. Variation in size spectra slopes is driven by variation in body size distributions and body size is in turn altered by 
temperature, either through reductions in taxon-specific body size, species turnover, or through changes in community structure (Bergmann 1847; Atkinson 1994; Daufresne et al. 2009; Winderet al. 2009; O'Gorman et al. 2012). Thus, it is widely expected size-spectra slopes should vary across temperature gradients, though the direction of change is uncertain (Daufresne et al.2009; Dossena et al. 2012; O'Gorman et al. 2012). Theory predicts that warm environments should favor smaller individuals, and this is supported by empirical (James 1970; Atkinson 1994; Daufresneet al. 2009) and experimental observations (Yvon-Durocher et al. 2011; Dossena et al. 2012). However, O'Gormanet al. (2017) found that warmed Icelandic streams had shallower slopes, perhaps due to increased nutrient availability and changes in trophic transfer efficiency, leading to increased top-down effects of consumers on diatoms. In contrast, Dossena et al. (2012) found that slopes declined with temperature, but the effect varied over seasons. Mazurkiewicz et al. (2020) found no relationship between marine benthic size spectra and temperatures in arctic systems. These contrasting outcomes, derived from different experimental approaches, generate uncertainty in how size spectra slopes should scale with temperature across large spatial gradients. The results presented here support the hypothesis that slopes become steeper in response to increasing temperature.

However, while size spectra slopes scaled with temperature, the overall change was relatively small, with median slopes declining by only $\sim 0.1$ units across the temperature gradient. Direct comparisons of this effect size with previous studies of size spectra responses to temperature (e.g., Yvon-Durocher et al. 2012, O'Gorman et al. 2017) are hampered by the different approaches to estimating size spectra exponents (Edwards et al. 2017). However, in a 30-year dataset from the International Bottom Trawl Survey (ICES 2015), Edwards et al. (2020) found that size spectra slopes calculated using maximum likelihood varied $\sim 0.4$ units among years. In addition, among sample variation at NEON sites in this study was $\sim 0.3$ to 0.8 units, with measured slopes at one site varying from -1 to -1.8 among sample dates. Placing our results into this context, the influence of temperature appears small relative to variation due to other factors, including natural variation over time.

In contrast to the negative relationship of temperature with size-spectra slopes, community biomass was positively related to mean annual temperature. This is also in agreement with predicted effects of increasing environmental temperature supporting more small-bodied individuals. For example, an increase in community biomass could be driven by small-bodied individuals alone, if their increase was larger than the relative decrease in larger-bodied individuals. It is important to account for community biomass, as increased biomass at lower trophic levels may be able to support biomass at higher trophic levels (O'Gormanet al. 2012), even if trophic transfer efficiency is affected by temperatures (Trebilco et al. 2013). However, the magnitude of the random effects of site were large, and the effect of temperature was relatively small. Including additional predictor variables thought to affect community biomass, such as productivity or nutrient availability at the base of the food web (Morin et al.2001; Daan et al. 2005), may help explain additional variation.

Given the relatively small influence of temperature and the overlap in site-specific averages of size spectra (Figure 3A), our results provide an opportunity to use our range of size-spectra as a baseline indicator in studies of disturbance. Size-spectra relationships have been proposed as a universal indicator of ecological health, with deviations from "natural" size spectra representing disturbed systems (Jennings \& Blanchard 2004; Petchey \& Belgrano 2010; Trebilco et al. 2013). Defining "natural" is difficult without accounting for variation among broad spatial and temporal scales. By accounting for the effect of temperature on size spectra slopes in relatively undisturbed systems across 50 degrees of latitude over three years, our results reveal bounds that could help to gauge the severity of size spectra change in response to disturbance. For example, one approach would be to compare size spectra from disturbed sites to the posterior predictive distribution of size-spectra at a similar site in our study, with deviations outside of the expected range of natural variation indicating the level of disturbance. This may represent a powerful tool for assessing ecological condition. Indeed, as NEON data continues to be collected, it will be possible to compare our predictions to size-spectra collected after intense disturbances, such as extremely high or low flow events, temperature anomalies due to climate change, wildfires, flow debris, etc. This represents an exciting opportunity to test responses to disturbances at higher levels of organization, which has typically been difficult or impossible due to the large logistical efforts needed to collect community-wide data across broad spatial scales. Furthermore, data on post-disturbance size spectra within the NEON sites will provide valuable information on community 
recovery, and the magnitude, direction, and expected duration of altered size spectra.

\section{Acknowledgments}

We thank CJ Perovich for thoughtful discussion, and IA Sutton for help with maximum likelihood methods. This work was funded by an NSF EAGER award (DEB \#1837233) to JSW.

\section{References}

Atkinson, D. (1994). Temperature and Organism Size-A Biological Law for Ectotherms? Advances in Ecological Research , 25, 1-58.

Benke, A.C., Arsdall, T.C.V., Gillespie, D.M. \& Parrish, F.K. (1984). Invertebrate Productivity in a Subtropical Blackwater River: The Importance of Habitat and Life History. Ecological Monographs , 54, 25-63.

Bergmann, C. (1847). Über die Verhältnisse der Wärmeökonomie der Thiere zu ihrer Größe. Gottinger Studien , 3, 595-708.

Blanchard, J.L., Heneghan, R.F., Everett, J.D., Trebilco, R. \& Richardson, A.J. (2017). From bacteria to whales: using functional size spectra to model marine ecosystems. Trends in Ecology 83 Evolution , 32, $174-186$.

Blanchard, J.L., Jennings, S., Law, R., Castle, M.D., McCloghrie, P., Rochet, M.-J., et al. (2009). How does abundance scale with body size in coupled size-structured food webs? Journal of Animal Ecology , 78, $270-280$.

Brose, U., Dunne, J.A., Montoya, J.M., Petchey, O.L., Schneider, F.D. \& Jacob, U. (2012). Climate change in size-structured ecosystems.Philosophical Transactions of the Royal Society B: Biological Sciences , 367, 2903-2912.

Brown, J.H., Gillooly, J.F., Allen, A.P., Savage, V.M. \& West, G.B. (2004). Toward a metabolic theory of ecology. Ecology, 85, 1771-1789.

Bürkner, P.-C. (2018). Advanced Bayesian multilevel modeling with the R package brms. The R Journal, $10,395-411$.

Chesney, T. (2019). NEON User Guide to Aquatic Macroinvertebrate Collection (NEON.DP1.20120) .

Cross, W.F., Hood, J.M., Benstead, J.P., Huryn, A.D. \& Nelson, D. (2015). Interactions between temperature and nutrients across levels of ecological organization. Global Change Biology , 21, 1025-1040.

Daan, N., Gislason, H., Pope, J.G. \& Rice, J.C. (2005). Changes in the North Sea fish community: evidence of indirect effects of fishing? ICES Journal of Marine Science , 62, 177-188.

Daufresne, M., Lengfellner, K. \& Sommer, U. (2009). Global warming benefits the small in aquatic ecosystems. Proceedings of the National Academy of Sciences , 106, 6.

Dietze, M.C. (2017). Ecological Forecasting . Princeton University Press.

Dossena, M., Yvon-Durocher, G., Grey, J., Montoya, J.M., Perkins, D.M., Trimmer, M., et al. (2012). Warming alters community size structure and ecosystem functioning. Proceedings of the Royal Society B , 279, 3011-3019.

Edwards, A.M., Robinson, J.P.W., Plank, M.J., Baum, J.K. \& Blanchard, J.L. (2017). Testing and recommending methods for fitting size spectra to data. Methods in Ecology and Evolution , 8, 57-67.

Gabry, J., Simpson, D., Vehtari, A., Betancourt, M., \& Gelman, A. (2019). Visualization in Bayesian workflow. Journal of the Royal Statistical Society: Series A (Statistics in Society), 182, 389-402.

Gardner, J.L., Peters, A., Kearney, M.R., Joseph, L. \& Heinsohn, R. (2011). Declining body size: a third universal response to warming? Trends in Ecology \& Evolution , 26, 285-291. 
Gelman, A. \& Rubin, D.B. (1992). Inference from iterative simulation using multiple sequences. Statistical Science, , 7, 457-511.

Grimm, N.B. (1988). Role of Macroinvertebrates in Nitrogen Dynamics of a Desert Stream. Ecology , 69, 1884-1893.

Hobbs, N.T. \& Hooten, M.B. (2015). Bayesian Models: A Statistical Primer for Ecologists . Princeton University Press, rinceton, New Jersey.

James, F.C. (1970). Geographic Size Variation in Birds and Its Relationship to Climate. Ecology, 51, 365390.

Jennings, S. \& Blanchard, J.L. (2004). Fish abundance with no fishing: predictions based on macroecological theory. Journal of Animal Ecology , 73, 632-642.

Martínez, A., Larrañaga, A., Miguélez, A., Yvon-Durocher, G. \& Pozo, J. (2016). Land use change affects macroinvertebrate community size spectrum in streams: the case of Pinus radiata plantations.Freshwater Biology , 61, 69-79.

Mazurkiewicz, M., Górska, B., Renaud, P.E., Legeżyńska, J., Berge, J. \& Włodarska-Kowalczuk, M. (2019). Seasonal constancy (summer vs. winter) of benthic size spectra in an Arctic fjord. Polar Biol , 42, 1255-1270.

Mazurkiewicz, M., Górska, B., Renaud, P.E. \& Włodarska-Kowalczuk, M. (2020). Latitudinal consistency of biomass size spectra - benthic resilience despite environmental, taxonomic and functional trait variability. Sci Rep , 10, 4164.

McElreath, R. (2020). Statistical Rethinking: ABayesian Course with Examples in R and Stan . CRC press.

McGarvey, D.J. \& Kirk, A.J. (2018). Seasonal comparison of community-level size-spectra in southern coalfield streams of West Virginia (USA). Hydrobiologia , 809, 65-77.

Morin, A., Bourassa, N. \& Cattaneo, A. (2001). Use of size spectra and empirical models to evaluate trophic relationships in streams.Limnology and Oceanography , 46, 935-940.

National Ecological Observatory Network. (2020). Data Product DP1.20120.001, Macroinvertebrate collection. Provisional data downloaded from http://data.neonscience.org . Batelle, Boulder, CO USA, NEON.

O'Gorman, E.J., Pichler, D.E., Adams, G., Benstead, J.P., Cohen, H., Craig, N., et al. (2012). Impacts of warming on the structure and functioning of aquatic communities. Individual- to ecosystem-level responses. Advances in Ecological Research , 47, 81-176.

O'Gorman, E.J., Zhao, L., Pichler, D.E., Adams, G., Friberg, N., Rall, B.C., et al. (2017). Unexpected changes in community size structure in a natural warming experiment. Nature Climate Change , 7, 659-663.

Perkins, D.M., Durance, I., Edwards, F.K., Grey, J., Hildrew, A.G., Jackson, M., et al. (2018). Bending the rules: exploitation of allochthonous resources by a top-predator modifies size-abundance scaling in stream food webs. Ecol. Lett.

Petchey, O.L. \& Belgrano, A. (2010). Body-size distributions and size-spectra: universal indicators of ecological status? Biology Letters , 6, 434-437.

Pomeranz, J.P.F., Warburton, H.J. \& Harding, J.S. (2019). Anthropogenic mining alters macroinvertebrate size spectra in streams.Freshwater Biology , 64, 81-92.

R Development Core Team. (2017). R: A language and environment for statistical computing . R Core Team, Vienna, Austria.

Sprules \& Barth. (2015). Surfing the biomass size spectrum: some remarks on history, theory, and application. Canadian Journal of Fisheries and Aquatic Sciences, 73, 477-495.

Stan Development Team. (2018). RStan: the R interface to Stan . 
Trebilco, R., Baum, J.K., Salomon, A.K. \& Dulvy, N.K. (2013). Ecosystem ecology: size-based constraints on the pyramids of life. Trends in Ecology \& Evolution , 28, 423-431.

Warmbold, J.W. \& Wesner, J.S. (2018). Predator foraging strategy mediates the effects of predators on local and emigrating prey.Oikos , 127, 579-589.

White, E.P., Enquist, B.J. \& Green, J.L. (2008). On estimating the exponent of power-law frequency distributions. Ecology, 89, 905-912.

White, E.P., Ernest, S.K.M., Kerkhoff, A.J. \& Enquist, B.J. (2007). Relationships between body size and abundance in ecology. Trends in Ecology $\&$ Evolution, 22, 323-330.

Winder, M., Reuter, J.E. \& Schladow, S.G. (2009). Lake warming favours small-sized planktonic diatom species. Proceedings of the Royal Society B: Biological Sciences , 276, 427-435.

Woodward, G., Ebenman, B., Emmerson, M., Montoya, J.M., Olesen, J.M., Valido, A., et al. (2005). Body size in ecological networks. Trends in Ecology 85 Evolution , 7, 402-409.

Yvon-Durocher, G. \& Allen, A.P. (2012). Linking community size structure and ecosystem functioning using metabolic theory.Philosophical Transactions of the Royal Society B: Biological Sciences , 367, 2998-3007.

Yvon-Durocher, G., Montoya, J.M., Trimmer, M. \& Woodward, G. (2011). Warming alters the size spectrum and shifts the distribution of biomass in freshwater ecosystems. Global Change Biology , 17, 1681-1694.

Tables and Figures

Table 1. Site-level information for stream sites in the National Ecological Observatory Network.

\section{Hosted file}

image1.emf available at https://authorea.com/users/367796/articles/487073-spatiotemporalvariability-of-abundance-size-spectra-in-streams-across-north-america

Table 2. Population-level coefficient estimates and $95 \%$ credible intervals for the effects of degree days on size spectra slopes ( $b$ exponent) and community biomass.

\begin{tabular}{llllll}
\hline Model & Coefficient & Estimate & Q2.5 & Q97.5 & R-hat \\
\hline$b$ exponent & y-intercept & -1.2 & -1.3 & -1.1 & 1 \\
& degree days & -0.003 & -0.007 & 0.0004 & 1 \\
Biomass & y-intercept & 6.7 & 6.2 & 7.5 & 1 \\
& degree days & 0.04 & -0.008 & 0.08 & 1 \\
\hline
\end{tabular}




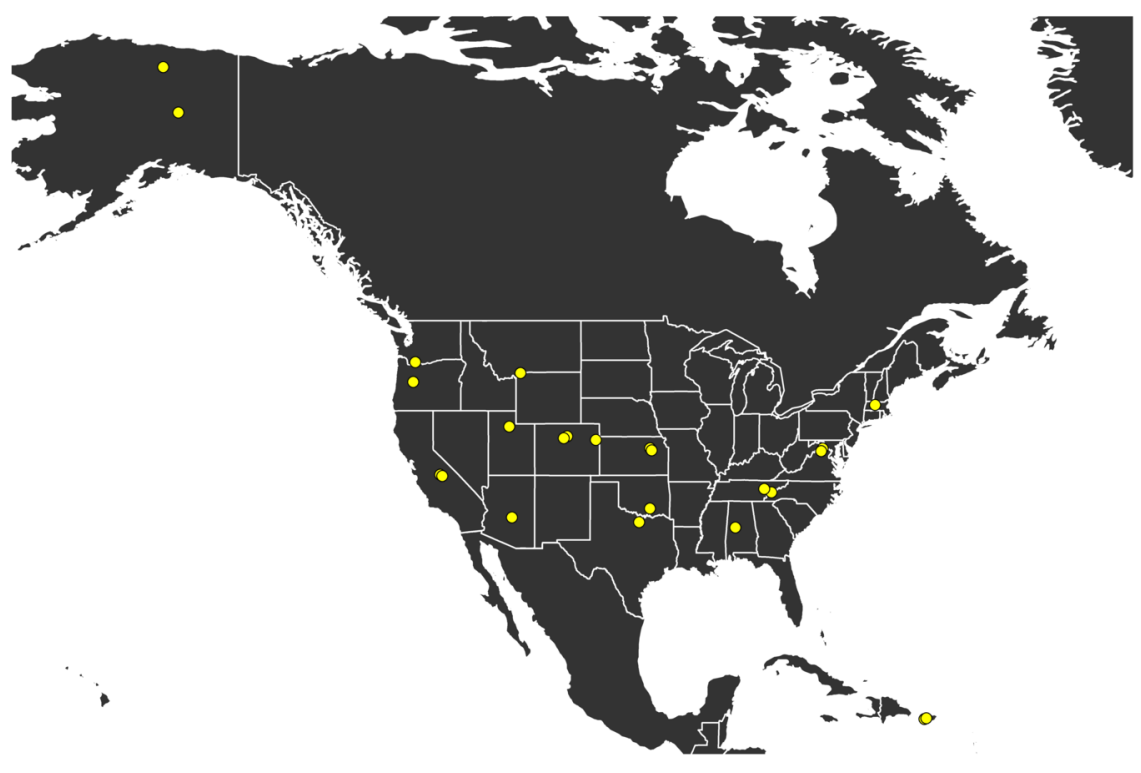

Figure 1. Map of site locations across North America.
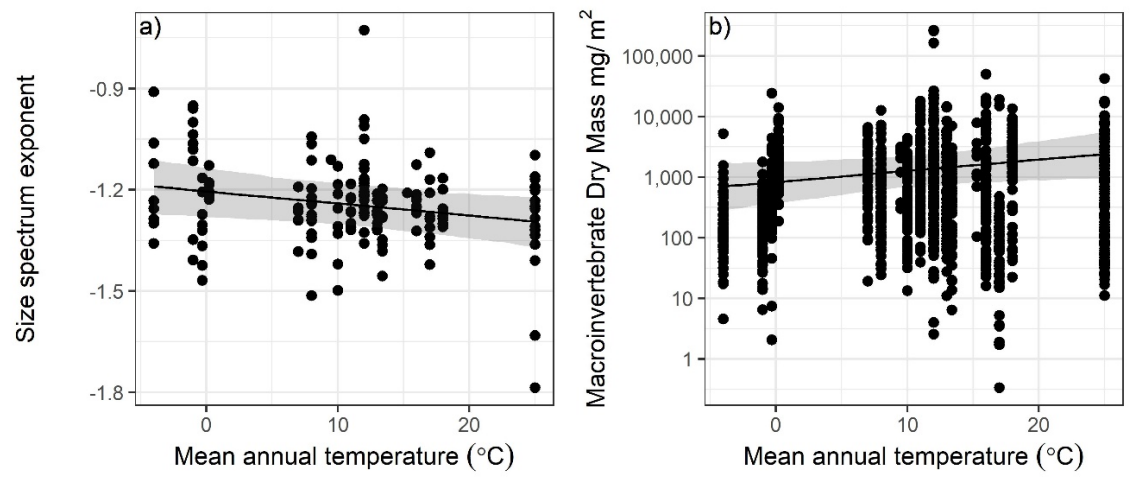

Figure 2 . Effect of mean annual temperature on the slope and intercept of normalized size-spectra relationships. Horizontal lines and shading indicate the median and $95 \%$ CrI of the change in slopes or intercepts across degree day values. Dots indicate individual collections. 

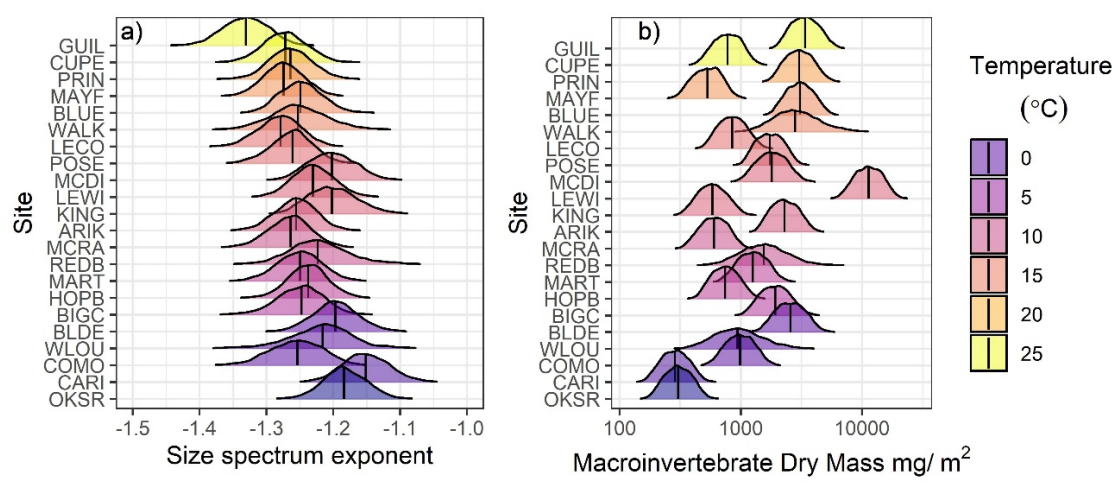

Figure 3. Posterior distributions of slope (a) and biomass (b) across sites where color corresponds to mean annual temperature. Black vertical lines within density distributions show the median value for a given collection. Sites are ordered by decreasing mean annual temperature, e.g., GUIL is the warmest site at $25^{\circ} \mathrm{C}$, while OKSR is the coldest at $-4^{\circ} \mathrm{C}$. 